\title{
Author Correction: Brain leaks and consumer neurotechnology
}

Marcello lenca, Pim Haselager and Ezekiel J. Emanuel

Correction to: Nature Biotechnology https://doi.org/10.1038/nbt.4240, published online 6 September 2018.

In the version of this article initially published, StarStim8 was misspelled StartSim8 and Flappy Whale was described as using EEG and MEG; it uses only EEG. The legend to Table 1 read "CES, cranial electrotherapy stimulation; PCS, transcranial pulsed current stimulation; TNS, trigeminal nerve stimulation"; the correct legend is "CES, cranial electrotherapy stimulation; ECG, electrocardiography; EMG, electromyography; TENS, transcutaneous electrical nerve stimulation; TNS, trigeminal nerve stimulation; tPCS, transcranial pulsed current stimulation; tRCS, transcranial rippled current stimulation." The table also indicated that the Ultracortex 'Mark IV' EEG Headset uses EEG, MEG and ECG; that the Cefaly uses external tRNS; and that the Thync uses tDCS; in fact, the Ultracortex uses EEG, EMG and ECG; the Cefaly uses external TNS; and the Thync uses tDCS and TENS. The manufacturer of the Cefaly was given as Roxon (Canada); the correct manufacturer is Cefaly Technology (Belgium). The table heading "Type of data collected" should have read "Type of data collected or stimulation." The errors have not been corrected in the original article. A corrected Table 1 appears here.

Table 1 | Neurotechnologies that are already marketed DTC

\begin{tabular}{|c|c|c|c|c|c|c|}
\hline Device name & Manufacturer & $\begin{array}{l}\text { Type of data } \\
\text { collected or } \\
\text { stimulation }\end{array}$ & Connectivity & Price $^{\star}$ & Advertised uses & $\begin{array}{l}\text { Ethical } \\
\text { concerns }\end{array}$ \\
\hline \multicolumn{7}{|c|}{ Neuromonitoring headsets } \\
\hline Muse & InteraXon (Canada) & EEG & Wireless & $\$ 249.00$ & $\begin{array}{l}\text { Elevating meditation } \\
\text { experience }\end{array}$ & \multirow{3}{*}{$\begin{array}{l}\text { Privacy, data } \\
\text { security, } \\
\text { informed } \\
\text { consent, } \\
\text { reliability }\end{array}$} \\
\hline Insight & Emotiv Systems & EEG & Wireless & $\$ 299.00$ & $\begin{array}{l}\text { Self-assessment, cognitive } \\
\text { training, research, device } \\
\text { control }\end{array}$ & \\
\hline Epoc+ & Emotiv Systems & EEG & Wireless & $\$ 799.00$ & $\begin{array}{l}\text { Self-assessment, cognitive } \\
\text { training, research, device } \\
\text { control }\end{array}$ & \\
\hline Necomimi & Neurowear (Japan) & EEG & Wireless & $\$ 49.00$ & $\begin{array}{l}\text { Augmentation, device } \\
\text { control }\end{array}$ & \\
\hline $\begin{array}{l}\text { MindWave } \\
\text { Mobile } 2\end{array}$ & Neurosky & EEG & Wireless & $\$ 99.99$ & $\begin{array}{l}\text { Self-assessment, } \\
\text { meditation, gaming, device } \\
\text { control }\end{array}$ & \\
\hline $\begin{array}{l}\text { Ultracortex } \\
\text { 'Mark IV' EEG } \\
\text { Headset }\end{array}$ & Open $\mathrm{BCl}$ & $\mathrm{EEG}, \mathrm{EMG}, \mathrm{ECG}$ & Wired & $\begin{array}{l}\$ 499.99-599.99 \\
\text { (unassembled) }\end{array}$ & $\begin{array}{l}\text { Self-assessment, cognitive } \\
\text { training, research, open } \\
\text { development }\end{array}$ & \\
\hline $\begin{array}{l}\text { Sleep Shepherd } \\
\text { Blue }\end{array}$ & Sleep Shepherd & $\begin{array}{l}\text { EEG (with binaural } \\
\text { beat biofeedback) }\end{array}$ & Wireless & $\$ 199.99$ & Improved sleep efficiency & \\
\hline \multicolumn{7}{|c|}{ Neuromodulation tools } \\
\hline GoFlow & Foc.Us (US/UK) & tDCS & Wired & $\$ 139.00$ & $\begin{array}{l}\text { Improved concentration, } \\
\text { brain training }\end{array}$ & \multirow{5}{*}{$\begin{array}{l}\text { Safety, } \\
\text { privacy, dat } \\
\text { security, } \\
\text { informed } \\
\text { consent, } \\
\text { reliability }\end{array}$} \\
\hline Focus V3 & Foc.Us (US/UK) & $\begin{array}{l}\text { tDCS, tRCS, tRNS, } \\
\text { tPCS, tACS }\end{array}$ & Wireless & $\$ 399.00$ & $\begin{array}{l}\text { Improved concentration, } \\
\text { brain training }\end{array}$ & \\
\hline Cefaly & $\begin{array}{l}\text { Cefaly Technology } \\
\text { (Belgium) }\end{array}$ & External TNS & Wireless & $\$ 349.00$ & $\begin{array}{l}\text { Treatment and prevention } \\
\text { of migraines }\end{array}$ & \\
\hline Thync & Thync Global & tDCS, TENS & Wireless & $\$ 150.00$ & $\begin{array}{l}\text { Improved sleep efficiency, } \\
\text { stress release }\end{array}$ & \\
\hline $\begin{array}{l}\text { Fisher Wallace } \\
\text { Stimulator }\end{array}$ & $\begin{array}{l}\text { Fisher Wallace } \\
\text { Laboratories }\end{array}$ & CES & Wired & $\$ 699.00$ & $\begin{array}{l}\text { Treatment of depression, } \\
\text { anxiety and insomnia }\end{array}$ & \\
\hline
\end{tabular}

*Price, as of May 20, 2018. CES, cranial electrotherapy stimulation; ECG, electrocardiography; EMG, electromyography; TENS, transcutaneous electrical nerve stimulation; TNS, trigeminal nerve stimulation; tPCS, transcranial pulsed current stimulation; tRCS, transcranial rippled current stimulation. 